PROCEEDINGS OF THE

AMERICAN MATHEMATICAL SOCIETY

Volume 126, Number 8, August 1998, Pages 2205-2211

S 0002-9939(98)04308-1

\title{
LINEAR OPERATORS \\ THAT PRESERVE MAXIMAL COLUMN RANKS OF NONNEGATIVE INTEGER MATRICES
}

\author{
SEOK-ZUN SONG
}

(Communicated by Lance W. Small)

\begin{abstract}
The maximal column rank of an $m$ by $n$ matrix over a semiring is the maximal number of the columns of $A$ which are linearly independent. We characterize the linear operators which preserve the maximal column ranks of nonnegative integer matrices.
\end{abstract}

\section{INTRODUCTION AND PRELIMINARIES}

A semiring is essentially a ring in which only the zero is required to have an additive inverse. Thus all rings are semirings. The nonnegative integers (with the usual arithmetic), $Z^{+}$, and the Boolean algebra of two elements are combinatorially interesting examples of semirings. Algebraic operations on matrices over a semiring and such notions as linearity and invertibility are also defined as if the underlying scalars were in a field.

The set of $m \times n$ matrices with entries in $Z^{+}$is denoted by $\mathbb{M}_{m, n}\left(Z^{+}\right)$.

A set of vectors ( $m \times 1$ matrices) is a semimodule [1] if it is closed under addition and scalar multiplication. A subset $\mathcal{W}$ of a semimodule $\mathcal{V}$ is a spanning set if each vector in $\mathcal{V}$ can be written as a sum of scalar multiples (i.e. a linear combination) of elements of $\mathcal{W}$.

The $m \times n$ matrix all of whose entries are zero except its $(i, j)$ th, which is 1 , is denoted $E_{i j}$. We call $E_{i j}$ a cell. The set of cells spans $\mathbb{M}_{m, n}\left(Z^{+}\right)$. Let $\mathbf{e}_{i}$ be the $n \times 1$ matrix with a " 1 " in the $i$ th position and zero elsewhere. We say that $A$ is a column matrix if $A=\mathbf{a e}_{i}^{t}$ for some $1 \leq i \leq n$ and some $\mathbf{a} \in \mathbb{M}_{m, 1}\left(Z^{+}\right)$.

The column space of a matrix $A \in \mathbb{M}_{m, n}\left(Z^{+}\right)$is the semimodule spanned by the columns of $A$ over $Z^{+}$. Since the column space is spanned by a finite set of vectors, it contains a spanning set of minimum cardinality; that cardinality is the column rank [2] of $A, \chi(A)$.

A set $G$ of vectors over $Z^{+}$is linearly dependent [2] if for some $g \in G, g$ is a linear combination of elements in $G-\{g\}$. Otherwise $G$ is linearly independent.

The maximal column rank [5], $\psi(A)$, of an $m \times n$ matrix $A \in \mathbb{M}_{m, n}\left(Z^{+}\right)$is the maximal number of the columns of $A$ which are linearly independent over $Z^{+}$.

Received by the editors June 4, 1996 and, in revised form, January 6, 1997.

1991 Mathematics Subject Classification. Primary 15A36, 15A03, 15A04.

Key words and phrases. Maximal column rank, linear operator.

The author wishes to acknowledge the financial support of the Korea Research Foundation made in the program year of 1997.

(C) 1998 American Mathematical Society 
It follows that

$$
0 \leq \chi(A) \leq \psi(A) \leq n
$$

for all $m \times n$ matrices $A$ over $Z^{+}$.

The inequality in (1.1) may be strict over $Z^{+}$. For example, we consider the matrix

$$
A=[1,2,3]
$$

over $Z^{+}$. Then the column rank of $A$ is one, while the maximal column rank of it is two since the last two columns of $A$ are linearly independent over $Z^{+}$.

Recently Hwang, Kim and Song [5] characterized the linear operators that preserve the maximal column rank of matrices over the binary Boolean algebra. They also compared the column rank and the maximal column rank for matrices over certain semirings, and found that, except for small values of $m$ and $n$, the two ranks did not agree in general. In particular, they obtained the following relations between column rank and maximal column rank over $\mathbb{M}_{m, n}\left(Z^{+}\right)$.

Theorem $1.1([5])$. Let $\alpha\left(Z^{+}, m, n\right)$ be the largest $k$ such that for all $m \times n m a$ trices $A$ over $Z^{+}, \chi(A)=\psi(A)$ if $\chi(A) \leq k$ and there is at least one $m \times n$ matrix $A$ over $Z^{+}$with $\chi(A)=k$. Then for $m \geq 1$,

$$
\alpha\left(Z^{+}, m, n\right)=\left\{\begin{array}{lll}
1 & \text { if } & n=1, \\
2 & \text { if } & n=2, \\
0 & \text { if } & n \geq 3 .
\end{array}\right.
$$

If $A$ is a matrix over $Z^{+}$and $A=\mathbf{u a}^{t}$, then $\mathbf{a}$ and $\mathbf{u}$ are called right and left factors of $A$ respectively.

Lemma 1.2. For $A \in \mathbb{M}_{m, n}\left(Z^{+}\right), \psi(A)=1$ if and only if $A$ can be factored as ua ${ }^{t}$ for some nonzero $\mathbf{u} \in \mathbb{M}_{m, 1}\left(Z^{+}\right)$and $\mathbf{a} \in \mathbb{M}_{m, 1}\left(Z^{+}\right)$with $\psi\left(\mathbf{a}^{t}\right)=1$.

Proof. If $\psi(A)=1$, then there exists one column $\mathbf{a}_{k}$ of $A$ such that all the other columns $\mathbf{a}_{i}$ are linearly dependent on each other, and hence all $\mathbf{a}_{i}$ are expressed as scalar multiples of $\mathbf{a}_{k}$, that is, $\mathbf{a}_{i}=\alpha_{i} \mathbf{a}_{k}$ for some $\alpha_{i} \in Z^{+}$. Therefore $A=$ $\mathbf{a}_{k}\left[\alpha_{1}, \cdots, \alpha_{n}\right]$.

Let $\mathbf{u}=\mathbf{a}_{k}, \mathbf{a}^{t}=\left[\alpha_{1}, \cdots, \alpha_{n}\right]$. Then the fact that $\psi\left(\mathbf{a}^{t}\right)=1$ follows from $\psi(A)=1$.

The converse is clear.

\section{LiNEAR OPERATORS THAT PRESERVE MAXIMAL COLUMN RANKS OVER $\mathbb{M}_{m, n}\left(Z^{+}\right)$}

A function $T$ mapping $\mathbb{M}_{m, n}\left(Z^{+}\right)$into itself is called an operator on $\mathbb{M}_{m, n}\left(Z^{+}\right)$. The operator $T$

1. is linear if $T(\alpha A+\beta B)=\alpha T(A)+\beta T(B)$ for all $\alpha, \beta \in Z^{+}$and all $A, B \in$ $\mathbb{M}_{m, n}\left(Z^{+}\right)$

2. preserves maximal column rank if $\psi(A)=\psi(T(A))$ for all $A \in \mathbb{M}_{m, n}\left(Z^{+}\right)$;

3. strongly preserves maximal column rank 1 provided that $\psi(T(A))=1$ if and only if $\psi(A)=1$ for all $A \in \mathbb{M}_{m, n}\left(Z^{+}\right)$.

In this section we obtain characterizations of the linear operators which preserve maximal column ranks of matrices over nonnegative integers. 
Lemma 2.1. Let $A=\mathbf{h}\left(\mathbf{e}_{1}\right)^{t}, B=\mathbf{h}\left(\mathbf{e}_{2}\right)^{t}$ be matrices in $\mathbb{M}_{m, n}\left(Z^{+}\right)$with $\mathbf{h} \in$ $\mathbb{M}_{m, 1}\left(Z^{+}\right)$. Suppose $T$ is a linear operator from $\mathbb{M}_{m, n}\left(Z^{+}\right)$into itself which strongly preserves maximal column rank 1. If $T(A)=\mathbf{u a}^{t}, T(B)=\mathbf{v b}^{t}$, where $\mathbf{a}^{t}=$ $\left[a_{1}, \cdots, a_{n}\right], \mathbf{b}^{t}=\left[b_{1}, \cdots, b_{n}\right]$ and $c \mathbf{u}=d \mathbf{v}$ for some nonzero $c, d \in Z^{+}$, then $a_{i} b_{i}=0$ for some $i$.

Proof. Suppose to the contrary that $a_{i} b_{i} \neq 0$ for all $i$. Since $c \mathbf{u}=d \mathbf{v}$, we have

$$
\begin{aligned}
d T(\alpha A+\beta B) & =\alpha d \mathbf{u} \mathbf{a}^{t}+\beta d \mathbf{v} \mathbf{b}^{t}=\alpha d \mathbf{u} \mathbf{a}^{t}+\beta c \mathbf{u} \mathbf{b}^{t} \\
& =\mathbf{u}(\alpha d \mathbf{a}+\beta c \mathbf{b})^{t} .
\end{aligned}
$$

By permuting columns, if necessary, we may assume that $b_{1} \leq b_{i}$ for all $i$ and $a_{1} \leq a_{j}$ for all $j$ such that $b_{j}=b_{1}$. Since $c$ and $d$ are nonzero, we have, for all sufficiently large $\beta$, that $d a_{1}+\beta b_{1} c \leq d a_{i}+\beta b_{i} c$ for all $i$. Since $d T(A+\beta B)$ has maximal column rank 1 for all $\beta$, any two columns are linearly dependent over $Z^{+}$. Thus, for any two columns, one is a scalar multiple of the other. Since $\psi(A+\beta B)=1$, from (2.1) we have

$$
\left(d a_{1}+\beta b_{1} c\right) \mid\left(d a_{i}+\beta b_{i} c\right)
$$

for sufficiently large $\beta$, and all $i$. Choose $k$ large and let $\beta=d k a_{1}$. Then

$$
\left(d a_{1}+d k a_{1} b_{1} c\right) \mid\left(d a_{i}+d k a_{1} b_{i} c\right)
$$

for all $i$ by (2.2). So $a_{1} \mid a_{i}$ for all $i$, and so we may assume that $a_{1}=1$. Since $a_{1}=1$ and $b_{1} \leq b_{i}, \alpha d+b_{1} c \leq \alpha d a_{i}+b_{i} c$ for all $\alpha$ and all $i$. Thus $\left(\alpha d+b_{1} c\right) \mid\left(\alpha d a_{i}+b_{i} c\right)$ for all $\alpha$ and all $i$, since $d T(\alpha A+B)$ has maximal column rank 1. Letting $\alpha=b_{1} c$, we have $\left(b_{1} c d+b_{1} c\right) \mid\left(b_{1} c d a_{i}+b_{i} c\right)$ for all $i$. It now follows that $b_{1} \mid b_{i}$ for all $i$, so we also may assume that $b_{1}=1$. Therefore

$$
(d+\beta c) \mid\left(d a_{i}+\beta b_{i} c\right)
$$

for all $\beta$ and all $i$ from (2.2).

Suppose that $a_{i} \neq b_{i}$ for some $i$. Say $a_{i}<b_{i}$. Letting $\beta=b_{i}$ and $\beta=b_{i}+1$, from (2.3) we have that for some $r, s \in Z^{+}$

$$
\begin{gathered}
d a_{i}+b_{i}^{2} c=r\left(d+b_{i} c\right), \\
d a_{i}+\left(b_{i}^{2}+b_{i}\right) c=s\left(d+\left(b_{i}+1\right) c\right),
\end{gathered}
$$

respectively. Subtracting (2.4) from (2.5), we have

$$
b_{i} c=(s-r)\left(d+b_{i} c\right)+s c
$$

If $s=r$, then from (2.6) we have $b_{i}=s$. So (2.4) gives

$$
d a_{i}+s^{2} c=s(d+s c)=s d+s^{2} c,
$$

that is, $a_{i}=s=b_{i}$, a contradiction since $a_{i}<b_{i}$. If $s>r$, then (2.6) gives

$$
b_{i} c=(s-r)\left(d+b_{i} c\right)+s c>b_{i} c,
$$

a contradiction. If $s<r$, then (2.6) gives

$$
b_{i} c=(s-r)\left(d+b_{i} c\right)+s c<s c .
$$

So $b_{i}<s<r$. From (2.4) we have

$$
d a_{i}+b_{i}^{2} c=r\left(d+b_{i} c\right)>s\left(d+b_{i} c\right)>s d+b_{i}^{2} c,
$$

that is, $d a_{i}>s d$. Thus we have $a_{i}>s>b_{i}$, which contradicts $a_{i}<b_{i}$.

For the case $b_{i}<a_{i}$, we also get contradictions by symmetric arguments. 
Thus $a_{i}=b_{i}$ for all $i$, that is, $\mathbf{a}=\mathbf{b}$. Then $\alpha A+\beta B$ has maximal column rank 2 for relatively prime positive integers $\alpha$ and $\beta$, since the first two columns of $\alpha A+\beta B$ are linearly independent. But

$$
\begin{aligned}
T(\alpha A+\beta B) & =\alpha T(A)+\beta T(B) \\
& =\alpha \mathbf{u a}^{t}+\beta \mathbf{v} \mathbf{b}^{t} \\
& =(\alpha \mathbf{u}+\beta \mathbf{v}) \mathbf{a}^{t}
\end{aligned}
$$

has maximal column rank 1 , since $\mathbf{a}^{t}$ has maximal column rank 1 from the construction. Hence we have a contradiction to the condition that $T$ strongly preserves maximal column rank 1 .

Lemma 2.2. Let $T$ be a linear operator from $\mathbb{M}_{m, n}\left(Z^{+}\right)$into itself. If $T$ strongly preserves maximal column rank 1 , then $T$ maps column matrices to column matrices.

Proof. Suppose to the contrary that $T$ maps a column matrix to a matrix which is not a column matrix. Say $X_{1}=\mathbf{x}\left(\mathbf{e}_{1}\right)^{t}$ and $T\left(X_{1}\right)$ has more than one nonzero column. For each $1 \leq i \leq n$, let $X_{i}=\mathbf{x}\left(\mathbf{e}_{i}\right)^{t}$. Let $S=\{1,2, \cdots, n\}$ and let $S_{1}=\{j$ : the $j$ th column of $T\left(X_{i}\right)$ is zero for all $\left.1 \leq i \leq n\right\}$. Then for each $i \in S-S_{1}$, there is a $j(i)$ such that the $i$ th column of $T\left(X_{j(i)}\right)$ is not zero. Now $T\left(X_{1}\right)$ has at least two nonzero columns, say columns $k_{1}$ and $k_{2}$. Let $S_{2}=S-S_{1}-\left\{k_{1}, k_{2}\right\}$, and let $A=X_{1}+\sum_{i \in S_{2}} X_{j(i)}$. Note that for any $k \in S-S_{1}$, the $k$ th column of $T(A)$ is nonzero. Further, since $A$ consists of at most $n-1$ distinct summands, each of which is a column matrix, there is at least one zero column in $A$, say the $i$ th. Let $B=X_{i}$. Since $T(A)$ has zero columns only corresponding to indices in $S_{1}$ (where $T(B)$ also must have a zero column), we can restrict our attention to those columns in $T(A)$ that are nonzero; hence we lose no generality in assuming that $T(A)$ has no zero column. Thus, since $A$, and hence $T(A)$, has maximal column rank $1, T(A)=\mathbf{u a}^{t}$, where $\mathbf{a}^{t}=\left[a_{1}, \cdots, a_{n}\right]$ has all nonzero entries which are linearly dependent, and some $u_{j} \neq 0$. Let $T(B)=\mathbf{v b}^{t}$ with $\mathbf{b}^{t}=\left[b_{1}, \cdots, b_{n}\right]$. Now we consider two cases:

Case 1. Assume that $c \mathbf{u} \neq d \mathbf{v}$ for all nonzero $c, d$ in $Z^{+}$. Since $\alpha A+B$ has maximal column rank 1 for any positive integer $\alpha$,

$$
T(\alpha A+B)=\left[\alpha a_{1} \mathbf{u}+b_{1} \mathbf{v}\left|\alpha a_{2} \mathbf{u}+b_{2} \mathbf{v}\right| \cdots \mid \alpha a_{n} \mathbf{u}+b_{n} \mathbf{v}\right]
$$

also has maximal column rank 1 . Thus we have, for some fixed $j$,

$$
\alpha a_{k} \mathbf{u}+b_{k} \mathbf{v}=\mu_{k}\left(\alpha a_{j} \mathbf{u}+b_{j} \mathbf{v}\right)
$$

for some positive integer, $\mu_{k}, k=1, \cdots, n$. If $a_{k} \neq \mu_{k} a_{j}$ for some $k$, then

$$
\alpha\left|a_{k}-\mu_{k} a_{j}\right| \mathbf{u}=\left|\mu_{k} b_{j}-b_{k}\right| \mathbf{v},
$$

which is a contradiction to the condition that $c \mathbf{u} \neq d \mathbf{v}$ for all nonzero $c, d$ in $Z^{+}$. Thus $a_{k}=\mu_{k} a_{j}$ and $b_{k}=\mu_{k} b_{j}, k=1, \cdots, n$. That is, $\mathbf{a}=a_{j} \mathbf{w}$ and $\mathbf{b}=b_{j} \mathbf{w}$, where $\mathbf{w}^{t}=\left[\mu_{1}, \cdots, \mu_{n}\right]$ with $\psi\left(\mathbf{w}^{t}\right)=\psi\left(\mathbf{a}^{t}\right)=1$. Then $\psi(T(\alpha A+\beta B))=$ $\psi\left(\left(\alpha a_{j} \mathbf{u}+\beta b_{j} \mathbf{v}\right) \mathbf{w}^{t}\right)=1$ for arbitrary $\alpha, \beta$ in $Z^{+}$. This contradicts the condition that $T$ strongly preserves maximal column rank 1 , since $\alpha A+\beta B$ has maximal column rank 2 for relatively prime $\alpha$ and $\beta$ in $Z^{+}$.

Case 2. Assume that $c \mathbf{u}=d \mathbf{v}$ for some nonzero $c, d$ in $Z^{+}$. Since $T(A)=\mathbf{u a}^{t}$ has maximal column rank 1 , all the columns of $\mathbf{a}^{t}$ are linearly dependent. So, without 
loss of generality, we can assume that $a_{1}=1$. For $T(B)=\mathbf{v b}^{t}$, we shall show that $b_{i} \neq 0$ for all $i$.

Suppose $b_{i}=0$ for some $i$. Choose $j$ such that $b_{j}=0$ and $a_{j} \leq a_{h}$ for all $h$ such that $b_{h}=0$. Since

$$
\begin{aligned}
d T(A+\beta B) & =d\left(\mathbf{u a}^{t}+\beta \mathbf{v} \mathbf{b}^{t}\right) \\
& =\mathbf{u}\left[d a_{1}+\beta c b_{1}, d a_{2}+\beta c b_{2}, \cdots, d a_{n}+\beta c b_{n}\right]
\end{aligned}
$$

has maximal column rank 1 for all $\beta$, choose $\beta$ such that $d a_{k}+\beta c b_{k}>d a_{j}$ for all $k$ with $b_{k} \neq 0$. Thus there exist distinct integers $\gamma, \delta$ such that, for fixed $k$ with $b_{k} \neq 0$,

$$
d a_{k}+\beta c b_{k}=\gamma d a_{j}
$$

and

$$
d a_{k}+(\beta+1) c b_{k}=\delta d a_{j} .
$$

Subtracting (2.7) from (2.8), we have

$$
c b_{k}=(\delta-\gamma) d a_{j} .
$$

Further, if $j \neq 1$, then $b_{1} \neq 0$. For, if $b_{1}=0$, then $a_{1}=1$ is the minimal entry in a, and hence $j=1$ from the construction of $a_{j}$. So, for $k=1, d a_{1}+\beta c b_{1}=\gamma_{1} d a_{j}$ from (2.6). Since $d a_{j} \mid c b_{1}$ from (2.9) for $k=1$, it follows that $d a_{j} \mid d a_{1}$, that is, $a_{j} \mid a_{1}(=1)$. Thus $a_{j}=1$. So we may assume that $j=1$.

Now,

$$
\begin{aligned}
c T(\alpha A+B) & =\left[c \alpha \mathbf{u}\left|c \alpha \mathbf{u} a_{2}+c \mathbf{v} b_{2}\right| \cdots \mid c \alpha \mathbf{u} a_{n}+c \mathbf{v} b_{n}\right] \\
& =\mathbf{v}\left[\alpha d, \alpha d a_{2}+c b_{2}, \cdots, \alpha d a_{n}+c b_{n}\right]
\end{aligned}
$$

must have maximal column rank 1 for all $\alpha$. Thus there are $\gamma_{i}$ such that $\alpha d a_{i}+c b_{i}=$ $\gamma_{i}(\alpha d)$ for all $i$.

It follows that $\alpha d \mid c b_{i}$ for all $\alpha$, and all $i=1, \cdots, n$, a contradiction since $b_{i} \neq 0$ for at least one $i$.

We now have shown that $b_{i} \neq 0$ for all $i$. Now, letting $A=X_{i}$ and $B=X_{j}, j=$ $1, \cdots, n$ and $j \neq i$, the above argument implies that $T(A)$ and $T(B)$ have no zero columns. This contradicts Lemma 2.1.

Hence the two cases show that $T$ maps column matrices to column matrices.

Theorem 2.3. Let $T: \mathbb{M}_{m, n}\left(Z^{+}\right) \rightarrow \mathbb{M}_{m, n}\left(Z^{+}\right)$be a linear operator. Then $T$ strongly preserves maximal column rank 1 if and only if there exist $Q \in \mathbb{M}_{m, m}\left(Z^{+}\right)$ which is nonsingular as a real matrix and a permutation matrix $P \in \mathbb{M}_{n, n}\left(Z^{+}\right)$ such that $T(A)=Q A P$ for all $A \in \mathbb{M}_{m, n}\left(Z^{+}\right)$.

Proof. Suppose there exist $Q$ and $P$ such that $T(A)=Q A P$ for all $A \in \mathbb{M}_{m, n}\left(Z^{+}\right)$ and $A$ has maximal column rank 1 . Then $A=\mathbf{x a}^{t}$ with $\psi\left(\mathbf{a}^{t}\right)=1$. That is, all the columns in $\mathbf{a}^{t}$ are linearly dependent on each other. Let $P^{t}$ correspond to a permutation $\pi \in S_{n}$. Then

$$
Q A P=Q \mathbf{x}\left(P^{t} \mathbf{a}\right)^{t}
$$

and the columns of $\left(P^{t} \mathbf{a}\right)^{t}$ are linearly dependent on each other. Hence $Q A P$ has maximal column rank 1. Further, assume that $Q A P$ has maximal column rank 1. Since $P$ is a permutation matrix in $\mathbb{M}_{n, n}\left(Z^{+}\right)$, multiplying $Q A P$ on the right by a permutation matrix $P^{-1}$ does not change the maximal column rank of $Q A P$. Hence $Q A$ has maximal column rank 1. Therefore all the columns $Q \mathbf{a}_{i}$ are linearly 
dependent, with $A=\left[\mathbf{a}_{1}, \cdots, \mathbf{a}_{n}\right]$. Thus for any two columns $Q \mathbf{a}_{k}$ and $Q \mathbf{a}_{h}$ of $Q A$ we have $Q \mathbf{a}_{k}=r_{k} Q \mathbf{a}_{h}$ or $Q \mathbf{a}_{h}=r_{h} Q \mathbf{a}_{h}$ with $r_{k}, r_{h} \in Z^{+}$. Hence $\mathbf{a}_{k}=r_{k} \mathbf{a}_{h}$ or $\mathbf{a}_{h}=r_{h} \mathbf{a}_{k}$ over the real field, and hence over $Z^{+}$since $Q$ is invertible as a real matrix. That is, $\psi(A)=1$. Thus $T$ strongly preserves maximal column rank 1 .

Conversely, suppose $T$ strongly preserves maximal column rank 1 . Let $X_{i}=$ $\mathbf{x}\left(\mathbf{e}_{i}\right)^{t}, i=1, \cdots, n$, for some fixed $\mathbf{x} \in\left(Z^{+}\right)^{m}$. By Lemma $2.2, T\left(X_{i}\right)=\mathbf{y}\left(\mathbf{e}_{\pi(i)}\right)^{t}$, where $\pi:\{1, \cdots, n\} \rightarrow\{1, \cdots, n\}$. If $\pi$ is not a permutation, then $\alpha T\left(X_{i}\right)+\beta T\left(X_{j}\right)$ has only one nonzero column for all $\alpha, \beta \in Z^{+}$. That is, $T\left(\alpha X_{i}+\beta X_{j}\right)$ has maximal column rank 1 for all $\alpha, \beta$, a contradiction since $\alpha X_{i}+\beta X_{j}$ has maximal column rank 2 for relatively prime $\alpha, \beta \in Z^{+}$. Thus $\pi$ is a permutation. So without loss of generality, we assume $\pi$ is the identity permutation, so that $T\left(X_{1}\right)=\mathbf{u}\left(\mathbf{e}_{1}\right)^{t}$ and $T\left(X_{2}\right)=\mathbf{v}\left(\mathbf{e}_{2}\right)^{t}$. If $u_{i} \neq 0$ and $v_{i}=0$, or vice versa, then $\mathbf{u}\left(\mathbf{e}_{1}\right)^{t}+\mathbf{v}\left(\mathbf{e}_{2}\right)^{t}$ has maximal column rank 2 , contradicting the condition that $T$ strongly preserves maximal column rank 1 since $X_{1}+X_{2}$ has maximal column rank 1 . Thus $u_{i}=0$ if and only if $v_{i}=0$. We assume without loss of generality that $0 \neq u_{1} \leq v_{1}$. Since $X_{1}+X_{2}$ has maximal column rank $1, \mathbf{v}=r \mathbf{u}$ for some $r \in Z^{+}$. If $r \neq 1$, choose $p$ relatively prime to $r$; then

$$
\begin{aligned}
T\left(p X_{1}+X_{2}\right) & =[p \mathbf{u}|\mathbf{v}| \mathbf{0}|\cdots| \mathbf{0}] \\
& =[p \mathbf{u}|r \mathbf{u}| \mathbf{0}|\cdots| \mathbf{0}]
\end{aligned}
$$

has maximal column rank 2 while $p X_{1}+X_{2}$ has maximal column rank 1 , a contradiction. Thus $r=1$. That is, $\mathbf{u}=\mathbf{v}$. It follows that $T\left(X_{i}\right)=\mathbf{u}\left(\mathbf{e}_{i}\right)^{t}$. In particular, when $X_{i}=E_{j i}$, there exists some vector $\mathbf{u}_{j}$ such that $T\left(E_{j i}\right)=\mathbf{u}_{j}\left(\mathbf{e}_{i}\right)^{t}$ for all $i, j$. Let $Q$ be the matrix $\left[\mathbf{u}_{1}\left|\mathbf{u}_{2}\right| \cdots \mid \mathbf{u}_{m}\right]$. Then for an arbitrary $A \in \mathbb{M}_{m, n}\left(Z^{+}\right)$,

$$
\begin{aligned}
T(A) & =\sum_{j=1}^{m} \sum_{i=1}^{n} a_{j i} T\left(E_{j i}\right) \\
& =\sum_{j=i}^{m} \sum_{i=1}^{n} a_{j i} \mathbf{u}_{j}\left(\mathbf{e}_{i}\right)^{t} .
\end{aligned}
$$

So the $(k, j)$ entry of $T(A)$ is $\sum_{i=1}^{m} a_{i j} u_{k i}$. The $(k, j)$ entry of $Q A$ is $\sum_{i=1}^{m} u_{k i} a_{i j}$, which is the $(k, j)$ entry of $T(A)$. Thus, $T(A)=Q A$ for all $A \in \mathbb{M}_{m, n}\left(Z^{+}\right)$.

Finally, we show that $Q$ is nonsingular as a real matrix. Suppose that $Q=\left(q_{i j}\right)$ is singular. Say, $Q \mathbf{x}=\mathbf{0}$ for some nonzero real vector $\mathbf{x}$. Since $\mathbf{x}$ can be considered as a solution of the homogeneous system of linear equations with coefficients $q_{i j} \in Z^{+}$, we may assume, without loss of generality, that the entries of $\mathbf{x}$ are all integers. So let $\alpha=1+\max _{1 \leq i \leq m}\left|x_{i}\right|$ and $\mathbf{z}=\alpha \mathbf{j}+\mathbf{x}$, where $\mathbf{j}$ is the vector of all 1's. Then $\mathbf{z} \in \mathbb{M}_{m, 1}\left(Z^{+}\right)$and $Q \mathbf{z}=Q(\alpha \mathbf{j}+\mathbf{x})=Q(\alpha \mathbf{j})$. Thus $T\left(\mathbf{z e}_{1}^{t}+\alpha \mathbf{j} \mathbf{e}_{2}^{t}\right)=$ $Q\left(\mathbf{z} \mathbf{e}_{1}^{t}\right)+Q\left(\alpha \mathbf{j} \mathbf{e}_{2}^{t}\right)=Q(\alpha \mathbf{j}) \mathbf{e}_{1}^{t}+Q(\alpha \mathbf{j}) \mathbf{e}_{2}^{t}=Q(\alpha \mathbf{j})\left(\mathbf{e}_{1}+\mathbf{e}_{2}\right)^{t}$ has maximal column $\operatorname{rank} 1$. Then $\mathbf{z}=k \alpha \mathbf{j}$, or $k \mathbf{z}=\alpha \mathbf{j}$, for some $k \in Z^{+}$. But then $Q \mathbf{z}=\mathbf{0}$ and hence $T\left(\mathbf{z e}_{1}^{t}\right)=\mathbf{0}$, contradicting the condition that $T$ strongly preserves maximal column rank 1. Thus $Q$ is nonsingular as a real matrix.

Corollary 2.4. A linear operator $T: \mathbb{M}_{m, n}\left(Z^{+}\right) \rightarrow \mathbb{M}_{m, n}\left(Z^{+}\right)$preserves maximal column rank if and only if there exist $Q \in \mathbb{M}_{m, m}\left(Z^{+}\right)$which is nonsingular as a real matrix and a permutation matrix $P \in \mathbb{M}_{n, n}\left(Z^{+}\right)$such that $T(A)=Q A P$ for all $A \in \mathbb{M}_{m, n}\left(Z^{+}\right)$.

Thus we have characterized the linear operators that preserve maximal column rank of nonnegative integer matrices. 


\section{ACKNOWLEDGEMENT}

The author would like to thank his parents (Pal-Bok Song, Yong-Bun Park) and his mother-in-law (Youn-Hee Seol) for their encouragements.

\section{REFERENCES}

[1] L.B.Beasley, S.J.Kirkland and B.L.Shader, Rank comparisons, Linear Algebra Appl. 221 (1995), 171-188. MR 96b:15001

[2] L.B.Beasley and N.J.Pullman, Semiring rank versus column rank, Linear Algebra Appl. 101 (1988), 33-48. MR 89e:15003

[3] L.B.Beasley and N.J.Pullman, Boolean rank-preserving operators and Boolean rank-1 spaces, Linear Algebra Appl. 59 (1984), 55-77. MR 85i:15004

[4] L.B.Beasley, D.A.Gregory and N.J.Pullman, Nonnegative rank-preserving operators, Linear Algebra Appl. 65 (1985), 207-223. MR 86b:15002

[5] S.G.Hwang, S.J.Kim and S.Z.Song, Linear operators that preserve maximal column rank of Boolean matrices, Linear and Multilinear Algebra 36 (1994), 305-313. MR 95b:15010

[6] S.Z.Song, Linear operators that preserve column rank of Boolean matrices, Proc. Amer. Math. Soc. 119 (1993), 1085-1088. MR 94a:15024

Department of Mathematics, Cheju National University, Cheju 690-756, Republic of Korea

E-mail address: szsong@cheju.cheju.ac.kr 